\title{
Design of Integrated Error Compensating System for the Portable Flexible CMMs
}

\author{
Qing-Song Cao, Jie Zhu, Zhi-Fan Gao, and Guo-Liang Xiong \\ College of Mechanical and Electrical Engineering, East China Jiaotong University, \\ Nanchang 330013, China
}

\begin{abstract}
During the working of portable flexible CMM, the mechanical arm's gravity and operating load generate dynamic strain, the thermal distortion also results in its length change. The caused errors would severely deteriorate the measuring accuracy. An error compensating system of the mechanical arm is designed in this paper, based on the monitor of dynamic strain and environmental temperature. The mathematical model of portable flexible coordinate measuring machine (CMM) was established.For the dynamic strain, four resistance strain gauges are sticked symmetrically on the root of each mechanical arm to monitor the dynamic strain. Then length error caused by the measuring arm's gravity or external operation load can be calculated through the analysis of stress. Strain compensation can be realized, when they are fed back to the measurement procedures. For thermal distortion, the temperature values acquired by the single-linear digital thermometer are substituted in the correction formula to calculate the relevant compensation dosage. Finally, these compensation dosages are feedbacked to the measuring program to have these errors compensated continually. The integrated error compensation system can improve the measurement accuracy of portable flexible CMM effectively.
\end{abstract}

Keywords: flexible arm, coordinates measuring machine, strain, temperature, error compensation.

\section{Introduction}

The traditional orthogonal coordinate measuring machines (CMMs) have the advantage of simple kinematical model and high measuring accuracy. However, with the development of the modernization of industry and manufacture, the limitations of traditional perpendicular CMMs also increasingly exposed to us, which can not meet the application requirements in many cases.

The flexible CMMs connect arm through revolting joint by turn, which is a new type of multiple degree and non-Cartesian system. It has the advantage of smaller volume, lighter weight, larger measuring range, portable and barrier-free measure compared with the traditional orthogonal CMMs. It is particularly suitable for the on-site mobile measure and it has gradually become the developing trend of the measurement field. However, due to its totally new structure, the mathematical modeling procedure is no longer simple on the one hand. On the other hand, the error of each joint will be seriously magnified by the measuring arm, and it will seriously affect the measurement accuracy. Therefore detecting and compensating for this error is very necessary. 
For flexible CMMs, the rotation angle of each joint, along with the mechanical parameters including length of each measuring arms and offset of every two joints etc, are substituted the kinematical model built by $\mathrm{D}-\mathrm{H}$ coordinate transform method. Then the measurement of 3-D coordinates can be realized. Therefore, the precision of 3-D coordinates is directly influenced by the angle error of each joint and the length error of every mechanical arm, to ensure the measuring accuracy error compensation system must be estblished.

Ye et al. $[1,2]$ considered error of the structural parameters during the machining and assembling process as the largest error source, and correct ideal model of the CMMs to get its error model. In this way, the measurement accuracy is greatly improved. Wang et al. [3] analyzed various factors that affect measurement results in detail, and consider that the processing and assemble error, the environmental temperature and strain, etc. affect the measurement precision of the CMMs. Wang [4] analyzed the impacts on the measuring results caused by external environment such as temperature, humidity and external power, and presented several measures that should be taken to reduce them.

\section{The Principle of Compensation for Integrated Error}

\subsection{Mathematical Model of the CMM}

Portable CMM adopts the whole serial revolted-joint structure, the relative position and posture between every two rods is the basis of coordinate measuring. And coordinate system of each rod should be built for the analysis of this relationship between them [5]. In 1955 Denavit and Hartenberg put forward the famous D-H homogeneous transformation method. By this method, 6 DOF coordinate system for structure schematic diagram of the CMM is built, as shown in Fig.1.

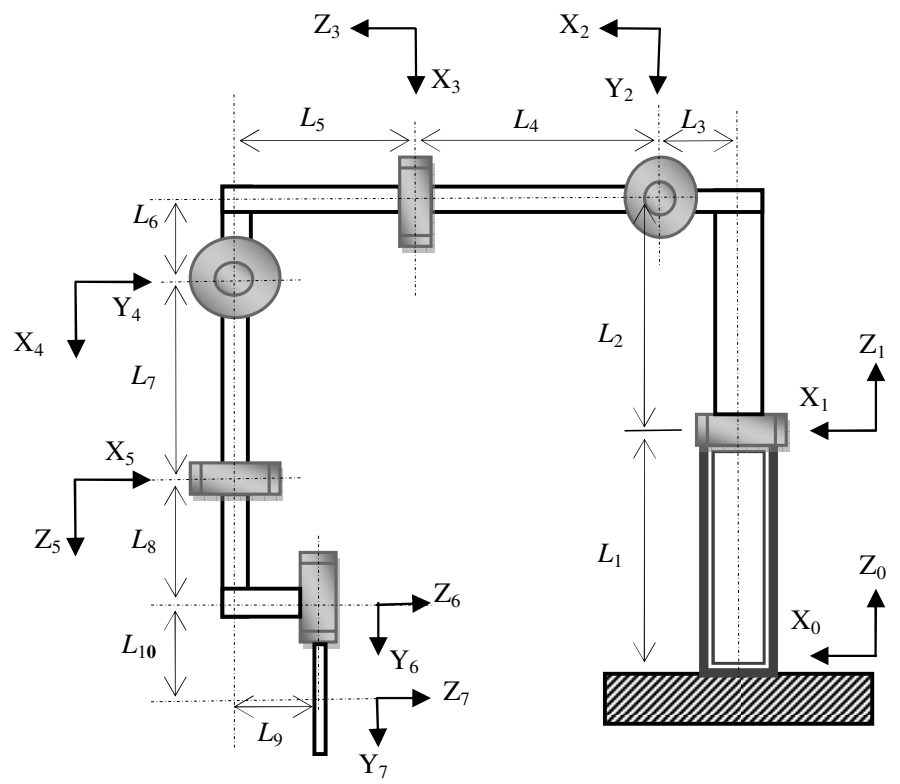

Fig. 1. Coordinates of the portable CMM 
The coordinate transformation matrix of coordinates from number $i+1$ to number $i$ is signed as $T_{i-1}{ }^{i}$. A CMM with $\mathrm{n}$ joints, the coordinate transformation matrixes of coordinate system are as below.

$$
T_{n}^{n-1}, T_{n-1}^{n-2}, \ldots, T_{2}^{1}, T_{1}^{0}
$$

If the position of one point in the final coordinate system is known, then coordinates of the measuring head in the base coordinate system can be obtained through continuous transformation of coordinate systems. On the basis of the coordinate systems built above and D-H coordinate transformation principle, the coordinate transformation matrix of the measuring head with respect to the base coordinate system can be expressed as

$$
A=T_{1}^{0} T_{2}^{1} T_{3}^{2} T_{4}^{3} T_{5}^{4} T_{6}^{5} T_{7}^{6}=\left[\begin{array}{cccc}
1 & 0 & 0 & 0 \\
0 & 1 & 0 & 0 \\
0 & 0 & 1 & L_{1} \\
0 & 0 & 0 & 1
\end{array}\right]
$$

$$
\begin{aligned}
& \\
& \left.\times \begin{array}{cccc}
\cos \theta_{1} & 0 & \sin \theta_{1} & L_{3} \cos \theta_{1} \\
\sin \theta_{1} & 1 & -\cos \theta_{1} & L_{3} \sin \theta_{1} \\
0 & 0 & 0 & L_{2} \\
0 & 0 & 0 & 1
\end{array}\right] \times\left[\begin{array}{cccc}
\cos \theta_{2} & 0 & \sin \theta_{2} & L_{4} \cos \theta_{2} \\
\sin \theta_{2} & 0 & -\cos \theta_{2} & L_{4} \sin \theta_{2} \\
0 & 1 & 0 & 0 \\
0 & 0 & 0 & 1
\end{array}\right] \\
& \times\left[\begin{array}{cccc}
\cos \theta_{3} & 0 & \sin \theta_{3} & L_{6} \cos \theta_{3} \\
\sin \theta_{3} & 0 & -\cos \theta_{3} & L_{6} \sin \theta_{3} \\
0 & 1 & 0 & L_{5} \\
0 & 0 & 0 & 1
\end{array}\right] \times\left[\begin{array}{cccc}
-\sin \theta_{4} & 0 & -\cos \theta_{4} & L_{7} \cos \theta_{4} \\
\cos \theta_{4} & 0 & -\sin \theta_{4} & L_{7} \sin \theta_{4} \\
0 & -1 & 0 & 0 \\
0 & 0 & 0 & 1
\end{array}\right] \\
& \times\left[\begin{array}{cccc}
-\sin \theta_{5} & 0 & \cos \theta_{5} & L_{9} \cos \theta_{5} \\
\cos \theta_{5} & 0 & \sin \theta_{5} & L_{9} \sin \theta_{5} \\
0 & 1 & 0 & L_{2} \\
0 & 0 & 0 & 1
\end{array}\right] \times\left[\begin{array}{cccc}
\cos \theta_{6} & 0 & \sin \theta_{6} & L_{10} \sin \theta_{6} \\
\sin \theta_{6} & 0 & \cos \theta_{6} & L_{10} \cos \theta_{6} \\
0 & 1 & 0 & 0 \\
0 & 0 & 0 & 1
\end{array}\right]
\end{aligned}
$$

As can be deduced from this mathematical model, the measurement result depend on the arms length $L_{\mathrm{i}}$ and the joint's rotating angle $\theta_{\mathrm{i}}$, the error of the arms length and the joint's rotating angle affected measuring accuracy directly.

\subsection{Principle of Compensation for Temperature Error}

During the measurement procedure, the environmental temperature should be kept as constant as possible. If not, the deviation of the temperature from the reference of $20^{\circ} \mathrm{C}$ will result in difference between real length and ideal length of each rod, which 
should not be neglected. For this kind of difference, temperature element is sticked to the rod to monitor its temperature, and then get it processed to get the relevant compensation dosage. The formula of the compensation dosage is

$$
\Delta L_{i}=(T-20) \times \alpha_{i}
$$

In this formula, $T$ is the environmental temperature, $\alpha_{i}$ is thermal expansion coefficient of the material. So when the affection of thermal variation on the measuring results is considered, and the compensation dosage is added to the ideal mathematical model, we can get the mathematical model with errors of temperature variation as follow

$$
A^{\prime}=T_{1}^{0} T_{2}^{1} T_{3}^{2} T_{4}^{3} T_{5}^{4} T_{6}^{5} T_{7}^{6}=\left[\begin{array}{cccc}
1 & 0 & 0 & 0 \\
0 & 1 & 0 & 0 \\
0 & 0 & 1 & L_{1}+\Delta L_{1} \\
0 & 0 & 0 & 1
\end{array}\right]
$$$$
\begin{aligned}
& \left.\times \begin{array}{cccc}
\cos \theta_{1} & 0 & \sin \theta_{1} & \left(L_{3}+\Delta L_{3}\right) \cos \theta_{1} \\
\sin \theta_{1} & 1 & -\cos \theta_{1} & \left(L_{3}+\Delta L_{3}\right) \sin \theta_{1} \\
0 & 0 & 0 & L_{2}+\Delta L_{2} \\
0 & 0 & 0 & 1
\end{array}\right] \times\left[\begin{array}{cccc}
\cos \theta_{2} & 0 & \sin \theta_{2} & \left(L_{4}+\Delta L_{4}\right) \cos \theta_{2} \\
\sin \theta_{2} & 0 & -\cos \theta_{2} & \left(L_{4}+\Delta L_{4}\right) \sin \theta_{2} \\
0 & 1 & 0 & 0 \\
0 & 0 & 0 & 1
\end{array}\right] \\
& \times\left[\begin{array}{cccc}
\cos \theta_{3} & 0 & \sin \theta_{3} & \left(L_{6}+\Delta L_{6}\right) \cos \theta_{3} \\
\sin \theta_{3} & 0 & -\cos \theta_{3} & \left(L_{6}+\Delta L_{6}\right) \sin \theta_{3} \\
0 & 1 & 0 & L_{5}+\Delta L_{5} \\
0 & 0 & 0 & 1
\end{array}\right] \times\left[\begin{array}{cccc}
-\sin \theta_{4} & 0 & -\cos \theta_{4} & \left(L_{7}+\Delta L_{7}\right) \cos \theta_{4} \\
\cos \theta_{4} & 0 & -\sin \theta_{4} & \left(L_{7}+\Delta L_{7}\right) \sin \theta_{4} \\
0 & -1 & 0 & 0 \\
0 & 0 & 0 & 1
\end{array}\right] \\
& \times\left[\begin{array}{cccc}
-\sin \theta_{5} & 0 & \cos \theta_{5} & \left(L_{9}+\Delta L_{9}\right) \cos \theta_{5} \\
\cos \theta_{5} & 0 & \sin \theta_{5} & \left(L_{9}+\Delta L_{9}\right) \sin \theta_{5} \\
0 & 1 & 0 & L_{2}+\Delta L_{2} \\
0 & 0 & 0 & 1
\end{array}\right] \times\left[\begin{array}{cccc}
\cos \theta_{6} & 0 & \sin \theta_{6} & \left(L_{10}+\Delta L_{10}\right) \sin \theta_{6} \\
\sin \theta_{6} & 0 & \cos \theta_{6} & \left(L_{10}+\Delta L_{10}\right) \cos \theta_{6} \\
0 & 1 & 0 & 0 \\
0 & 0 & 0 & 1
\end{array}\right]
\end{aligned}
$$

\subsection{Principle of Compensation for Strain Error}

In the measurement procedure, the bending deformation on measurement arm will result in the extra rotating angle of each joint. As the angle deviation would be seriously magnified through the arm, the impact caused must not be neglected.

The bending deformation caused by the operation force can be detected by strain gages sticked symmetrically around the measuring arm. The bridge type is most frequently used, which can amplify signal effectively. The circuit diagram for strain measurement is shown in fig. 2 . 


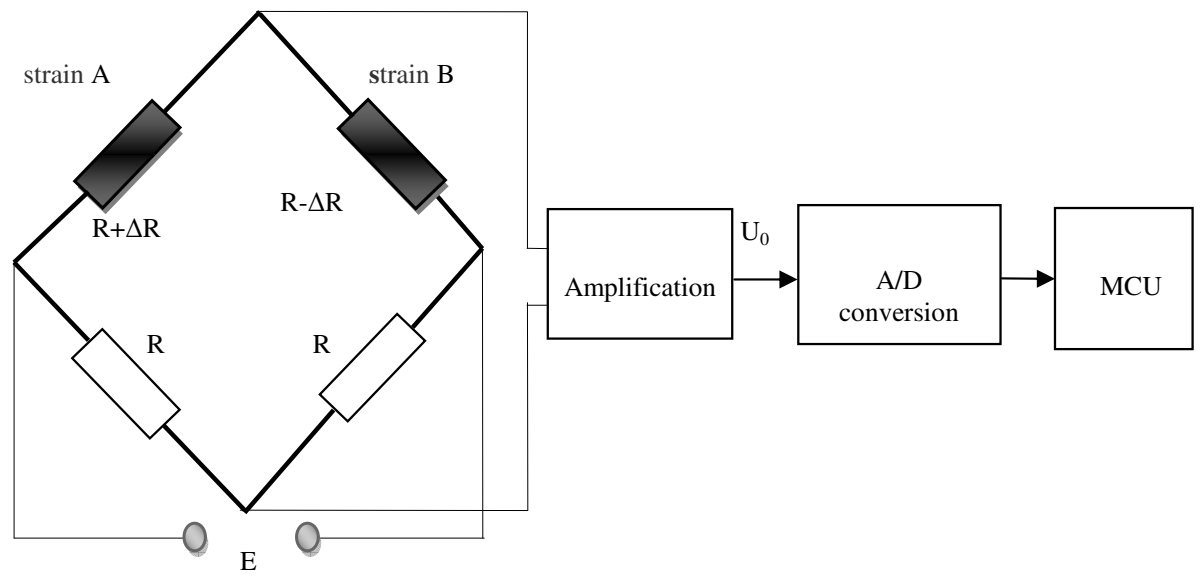

Fig. 2. Block diagram of strain-measuring circuit

As is shown in the figure, the bridge is in state of equilibrium, and there are no signals output when the measuring arm is not forced. Two strains are sticked on the adjacent bridge arms. The resistance of strain A gets bigger and that of strain $\mathrm{B}$ decreases when the arm is exposed to stress, the bridge is no longer balanced, and there is voltage signal output in this situation. As resistance strain is used in this paper, this signal is very weak, and it must be amplified first so that it can be treated afterwards. According to the above shows that can block, amplified output voltage of bridge road can be expressed as

$$
U_{0}=\frac{K_{0} \cdot K \cdot E \cdot \Delta L}{2 \cdot L}
$$

Where, $E$ is the input voltage, $K$ is the sensitivity coefficient of the strain gauge selected, $L$ is the length of the measuring arm. So the length variation of measuring arm can be obtained and expressed as

$$
\Delta L=\frac{2 \cdot U_{0} \cdot L}{E \cdot K \cdot K_{0}}
$$

And the corresponding extra rotation angle of the joint is

$$
\Delta \theta=\cos ^{-1}\left(\frac{L}{L+\Delta L}\right)=\cos ^{-1}\left(\frac{L \cdot E \cdot K \cdot K_{0}}{2 \cdot L \cdot U_{0}+L \cdot E \cdot K \cdot K_{0}}\right)
$$

Namely, this $\triangle \theta$ is the extra rotation angle of each joint caused by the measuring arm's own gravity or external operation force. And it is used as the compensation 
value and is substitute to mathematical model of the CMMs. So mathematical model with strain error can be expressed as

$$
\begin{aligned}
& A^{\prime \prime}=T_{1}^{0} T_{2}^{1} T_{3}^{2} T_{4}^{3} T_{5}^{4} T_{6}^{5} T_{7}^{6}=\left[\begin{array}{cccc}
1 & 0 & 0 & 0 \\
0 & 1 & 0 & 0 \\
0 & 0 & 1 & L_{1} \\
0 & 0 & 0 & 1
\end{array}\right] \\
& \times\left[\begin{array}{cccc}
\cos \left(\theta_{1}+\Delta \theta_{1}\right) & 0 & \sin \left(\theta_{1}+\Delta \theta_{1}\right) & L_{3} \cos \left(\theta_{1}+\Delta \theta_{1}\right) \\
\sin \left(\theta_{1}+\Delta \theta_{1}\right) & 1 & -\cos \left(\theta_{1}+\Delta \theta_{1}\right) & L_{3} \sin \left(\theta_{1}+\Delta \theta_{1}\right) \\
0 & 0 & 0 & L_{2} \\
0 & 0 & 0 & 1
\end{array}\right] \times\left[\begin{array}{cccc}
\cos \left(\theta_{2}+\Delta \theta_{2}\right) & 0 & \sin \left(\theta_{2}+\Delta \theta_{2}\right) & L_{4} \cos \left(\theta_{2}+\Delta \theta_{2}\right) \\
\sin \left(\theta_{2}+\Delta \theta_{2}\right) & 0 & -\cos \left(\theta_{2}+\Delta \theta_{2}\right) & L_{4} \sin \left(\theta_{2}+\Delta \theta_{2}\right) \\
0 & 1 & 0 & 0 \\
0 & 0 & 0 & 1
\end{array}\right] \\
& \times\left[\begin{array}{cccc}
\cos \left(\theta_{3}+\Delta \theta_{3}\right) & 0 & \sin \left(\theta_{3}+\Delta \theta_{3}\right) & L_{6} \cos \left(\theta_{3}+\Delta \theta_{3}\right) \\
\sin \left(\theta_{3}+\Delta \theta_{3}\right) & 0 & -\cos \left(\theta_{3}+\Delta \theta_{3}\right) & L_{6} \sin \left(\theta_{3}+\Delta \theta_{3}\right) \\
0 & 1 & 0 & L_{5} \\
0 & 0 & 0 & 1
\end{array}\right] \times\left[\begin{array}{cccc}
-\sin \left(\theta_{4}+\Delta \theta_{4}\right) & 0 & -\cos \left(\theta_{4}+\Delta \theta_{4}\right) & L_{7} \cos \left(\theta_{4}+\Delta \theta_{4}\right) \\
\cos \left(\theta_{4}+\Delta \theta_{4}\right) & 0 & -\sin \left(\theta_{4}+\Delta \theta_{4}\right) & L_{7} \sin \left(\theta_{4}+\Delta \theta_{4}\right) \\
0 & -1 & 0 & 0 \\
0 & 0 & 0 & 1
\end{array}\right] \\
& \times\left[\begin{array}{cccc}
-\sin \left(\theta_{5}+\Delta \theta_{5}\right) & 0 & \cos \left(\theta_{5}+\Delta \theta_{5}\right) & L_{9} \cos \left(\theta_{5}+\Delta \theta_{5}\right) \\
\cos \left(\theta_{5}+\Delta \theta_{5}\right) & 0 & \sin \left(\theta_{5}+\Delta \theta_{5}\right) & L_{9} \sin \left(\theta_{5}+\Delta \theta_{5}\right) \\
0 & 1 & 0 & L_{2} \\
0 & 0 & 0 & 1
\end{array}\right] \times\left[\begin{array}{cccc}
\cos \left(\theta_{6}+\Delta \theta_{6}\right) & 0 & \sin \left(\theta_{6}+\Delta \theta_{6}\right) & L_{10} \sin \left(\theta_{6}+\Delta \theta_{6}\right) \\
\sin \left(\theta_{6}+\Delta \theta_{6}\right) & 0 & \cos \left(\theta_{6}+\Delta \theta_{6}\right) & L_{10} \cos \left(\theta_{6}+\Delta \theta_{6}\right) \\
0 & 1 & 0 & 0 \\
0 & 0 & 0 & 1
\end{array}\right]
\end{aligned}
$$

\section{Design of the Compensation System for Integrated Errors}

\subsection{Overall Scheme}

To achieve compensation for errors caused by thermal change and strain, a compensation system is designed in this paper, which consists of the acquisition of temperature and strain, the calculation of compensation dosage and the modification of corresponding mathematical model. The overall diagram is shown in Figure 3.

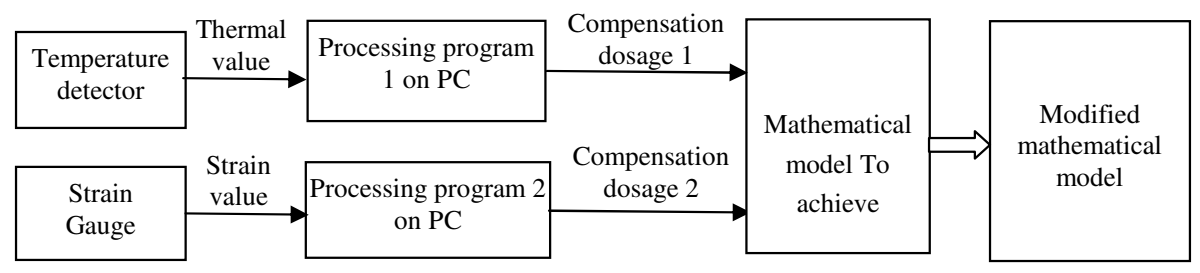

Fig. 3. Overall diagram of the error compensation system 


\subsection{Design of the Error Compensation System of Temperature}

As we know, most materials expand as the temperature increases and contract when they are cooled. The flexible arm CMMs are operated by people and are often used in workshop. The environmental temperature changes and the heat created by the electronic devices in the CMMs would result in length change of measuring arm. Therefore, measures have to be taken to modify these errors.

A new single-wire enhanced digital thermometer DS18B20 [6], which is produced by the DALLAS Corporation, is used in this paper. The measuring rang of temperature of this sensor is $-55 \sim 125^{\circ} \mathrm{C}$, and the measurement accuracy is $\pm 0.5^{\circ} \mathrm{C}$ among $-10 \sim 85^{\circ} \mathrm{C}$. As the sensor adopts a single-wire structure, the information can be sent or output by a single-wire interface, and the PC can read, write and convert the temperature through an I/O line. In addition, the sensor can achieve multiple points of temperature measurement, thus it can reduce the amount of line for temperature measurement [7]. The temperature measurement circuit is shown in figure 4.

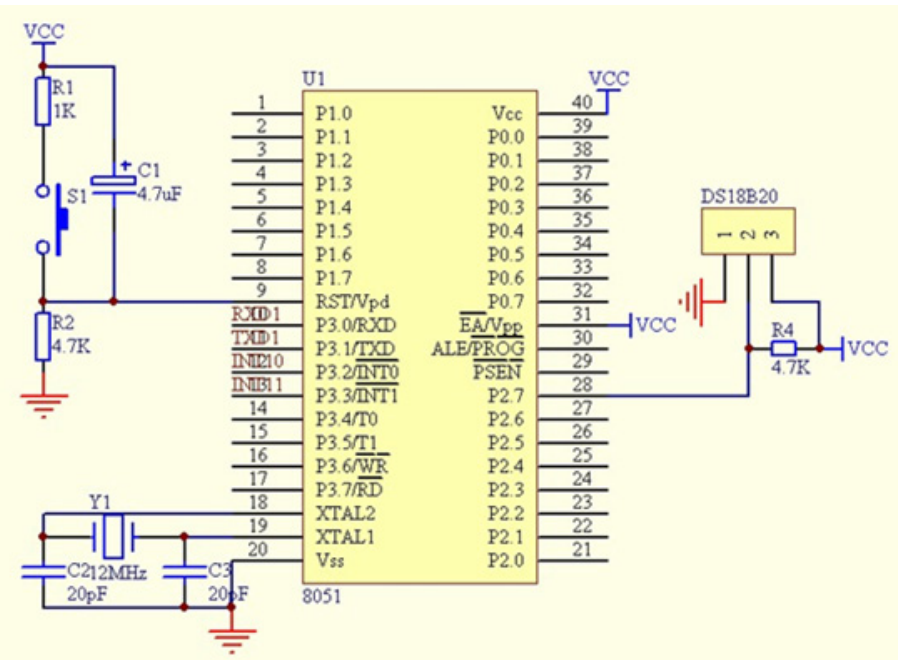

Fig. 4. Measurement circuit of temperature

The measuring circuit of thermal variation is very simple, and its function is mainly accomplished by software. The program diagram is shown in figure 5 . 


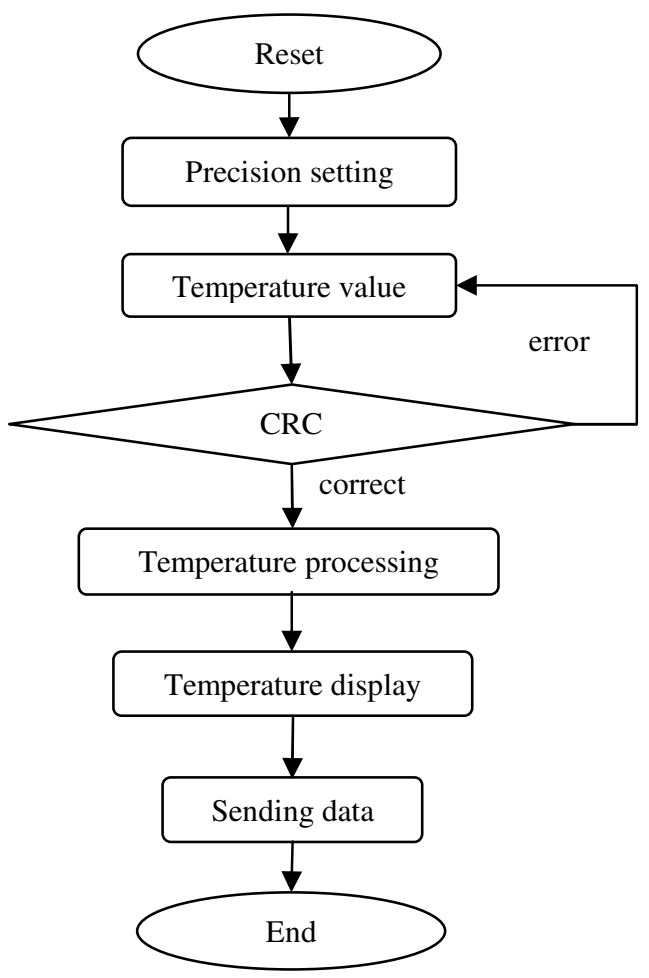

Fig. 5. Program diagram of thermal measurement

The temperature obtained from the single-wire digital thermometer DS18B20 is sent to computer through serial communication, and then it is compared with the ideal temperature of $20{ }^{\circ} \mathrm{C}$ to get the corresponding length change, using the thermal expansion coefficient of the selected material. On the PC, LabVIEW is used to obtain and calculate the compensation dosage of the corresponding temperature through serial communication. The program diagram is shown in figure 6 .

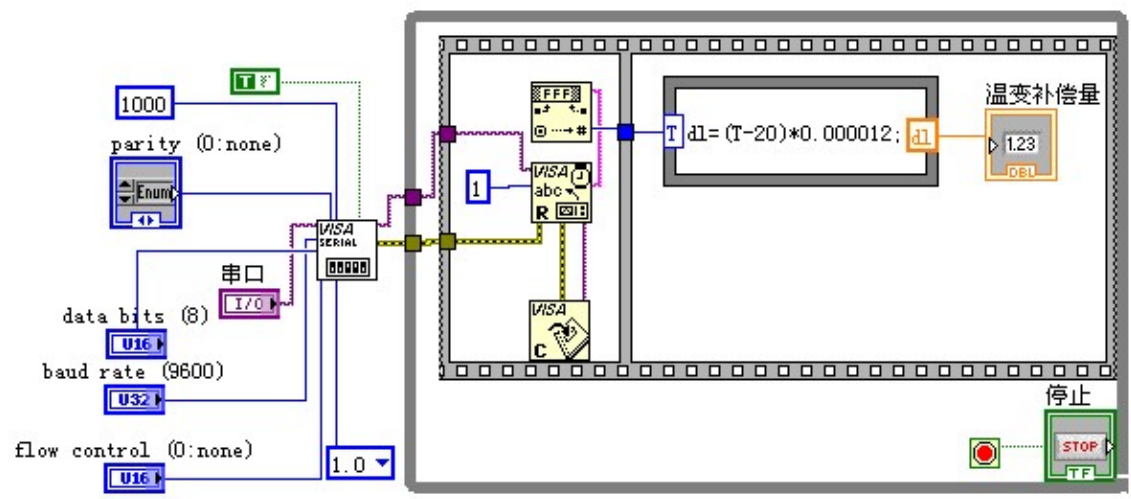

Fig. 6. Program diagram of thermal error compensation 


\subsection{Design of the Error Compensation System of Strain}

The arm of flexible CMM usually adopts long and thin circular pipe, so it is easy to bend and deform under its own gravity and the exterior operation power. Previous studies showed that it creates bending deformation of more than 10"at the two ends of the arm is very common. This is one of the most important factors limiting the measuring accuracy of this kind of CMMs.

The bending and deformation of CMM's each arm is not only in relation to the structural parameters of the arm, but also to the extension of the arm. It is difficult to calculate the amount of the bending and deformation of the arm precisely by theoretical calculation. So it is necessary to paste numbers of strain gauges to monitor deformation on each arm, and then make corresponding program to compensate for the errors immediately.

The bending and deformation of measuring arm is equal to increase the rotation angle of each joint additionally. Strain gauges are pasted symmetrically on the arm to monitor the bending and deformation along the axis in this paper. The strain gauges are linked as form of half bridge, and circuit of strain measurement is shown in figure 7 .

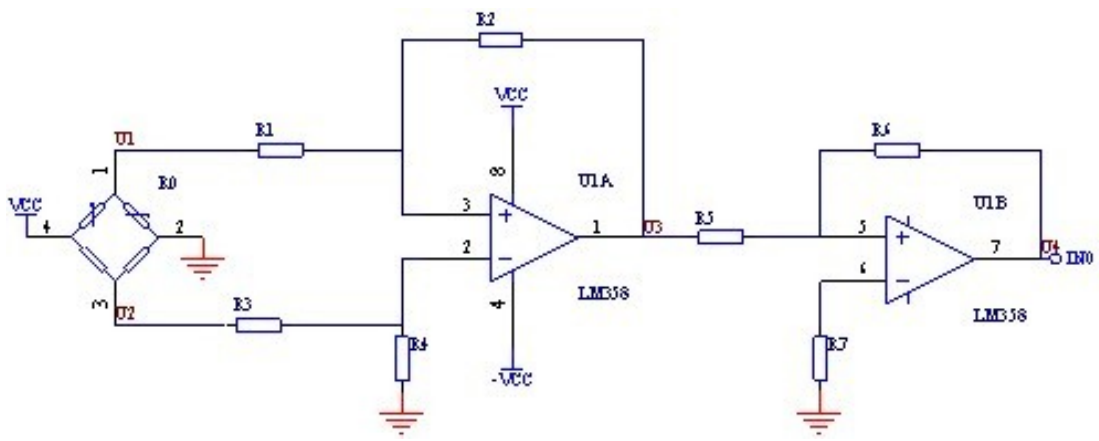

Fig. 7. Circuit of strain measurement

The amplified output voltage of the bridge is an analog quantity, and it must be converted to by $\mathrm{A} / \mathrm{D}$ converting circuit as the MCU can only obtain and deal with the digital quantity. The $\mathrm{A} / \mathrm{D}$ converting circuit is shown in figure 8 .

The programs compiled with LabVIEW on the PC for strain measurement is just like that of the temperature, we can replace the formula with the formulae 6 above. Take into account fully, the error of environmental thermal variation and dynamic strain was compiled with LabVIEW thus the Complete error compensation system was established. It is also the key technology to improve the measurement accuracy. 


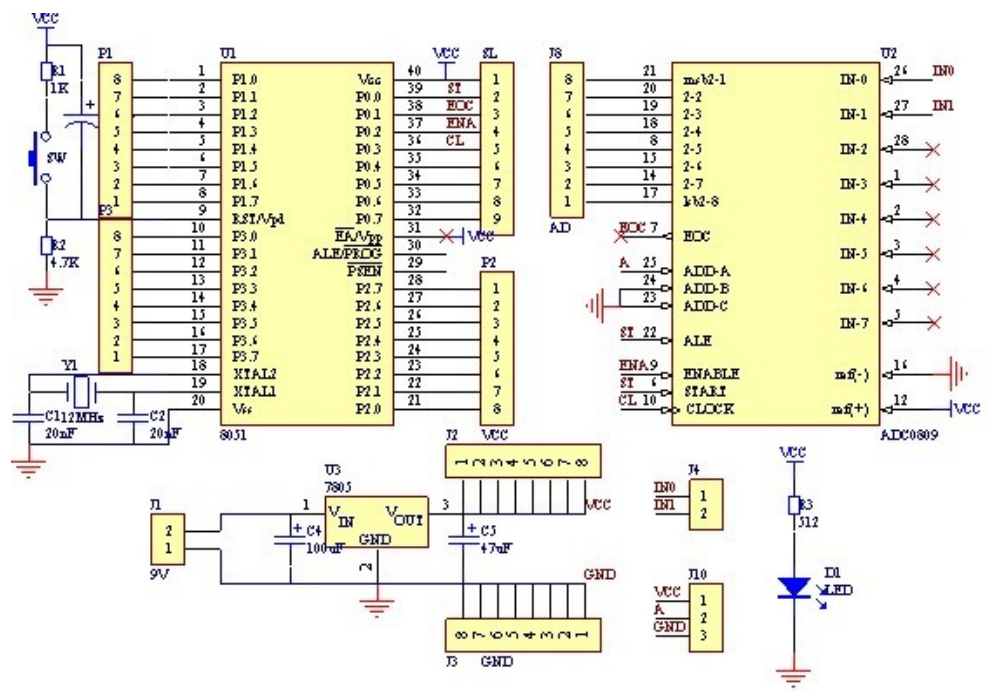

Fig. 8. A/D convertion circuit

\section{Conclusion}

Firstly, the ideal mathematical model of the portable CMM is established in this paper, and then the mathematical model with errors is established on the basis of analysis of parts of the error sources. At last an error compensation system based on environmental thermal variation and dynamic strain is designed from two aspects of hardware and software, the integrated error compensation system can improve the measurement accuracy of portable flexible CMM effectively.

\section{References}

1. Ye, D., Huang, Q. C., Che, R. S.: Error modeling for multi-joint coordinate measuring machine. J. Optics and Precision Engineering 7(2), 92-96 (1999)

2. Ye, D., Che, R.S.: Modeling and error analysis for Human-simulating multi-joint coordinate measuring machine. Journal of Harbin Institude of Technology 31(2), 28-32 (1999)

3. Wang, X.Y., Liu, S.G., Zhang, G.X.: Mathematical Model and error Analysis of the Articulated Arm Flexible CMM. J. Nanotechnology and Precission Engineering 3(4), 262-267 (2005)

4. Xu, J.X.: The develop of portable Articulated Arm CMM. D. Research institute of mechanical science, Beijing, (2007)

5. Li, Z.W., Wang, C.J., Zhou, X.H.: Mathematical Model and Parameter Calibration of the multi-joint coordinate measuring machine. J. Mechanical Processing Technology and Equipment (6), 35-36 (2006)

6. Denavit, J., Hartenberg, R.: A kinematic notation for lower pair mechanism based on matrices. J. ASME Journal of Applied Mechnics 22(6), 215-221 (1955)

7. Chen, T.: Application of Single Chip Processor and C language programming. China Machine Press, Beijing (2008) 